\title{
Análise físico funcional e cardiorrespiratória de pacientes em lista de espera para o transplante renal: estudo transversal
}

\author{
Physical, functional, and cardiorespiratory analysis of patients on the kidney \\ transplant waiting list: a cross-sectional study

\section{Daniela da Silva Martins', Paula Caitano Fontela' Maria Leocadia Bernardes do Amaral Padilha ${ }^{3}$ Eliane Roseli Winkelmann ${ }^{4}$}

\author{
'Universidade Regional do Noroeste do Estado do Rio Grande do Sul. liuí, Rio Grande do Sul, Brasil. \\ ORCID: 0000-0002-6035-2070. smartins.dani@yahoo.com.br \\ ${ }^{2}$ lijú, Rio Grande do Sul, Brasil. ORCID: 0000-0003-0086-7037. paula_fontella@hotmail.com \\ ${ }^{3}$ Hospital de Caridade de ljuí. lijú, Rio Grande do Sul, Brasil. ORCID: 0000-0001-5798-5515. Ipadilha@hci.org.br \\ ${ }^{4}$ Autora para correspondência. Universidade Regional do Noroeste do Estado do Rio Grande do Sul. liuí, Rio Grande do Sul, Brasil.
} ORCID: 0000-0002-4922-6516.elianew@unijui.edu.br

RESUMO I INTRODUÇÃO: Para o tratamento da doença renal crônica os pacientes necessitam de hemodiálise ou diálise peritoneal enquanto aguardam em lista de espera pelo transplante renal. Apesar dos avanços nos procedimentos de diálise, os pacientes que fazem esse tratamento apresentam baixa capacidade física e funcional e comorbidades que influenciam no tempo de espera. OBJETIVO: Descrever as características físicas, antropométricas e cardiorrespiratórias de pacientes em lista de espera para o transplante renal, assim como, analisar as variáveis de acordo com ○ tempo em lista de espera e tempo de diagnóstico da doença. MATERIAIS E MÉTODOS: Estudo transversal, descritivo e analítico, submetido e aprovado pelo Comitê de Ética em Pesquisa da UNIJUÍ parecer $\mathrm{n}^{\circ} 1.992 .013$. Foram avaliados 11 pacientes cadastrados no Hospital de Caridade de liuí, quanto aos dados sobre a doença renal e seu tratamento, tempo em lista de espera, fatores de risco cardiovasculares, resistência muscular de membros inferiores, força muscular respiratória e capacidade funcional submáxima. RESULTADOS: Foram avaliados 11 pacientes, sendo $54,5 \%$ do sexo feminino, média de idade de $51,9 \pm 11,4$ anos, $45,5 \%$ tinham doença renal de origem hipertensiva, tempo de diagnóstico da doença de $84,0 \quad(48,0-180,0)$ meses, $90,9 \%$ realizavam hemodiálise há uma mediana de $15,0(8,0-41,0)$ meses e estavam em lista de espera há $12,0(6,0-32,0)$ meses. Os fatores de risco cardiovasculares mais prevalentes foram hipertensão e sedentarismo. No teste de sentar e levantar em um minuto realizaram $17,0 \quad(16,0-22,0)$ repetições, pressão inspiratória máxima de $62,0(50,0-80,0) \mathrm{cmH}_{2} \mathrm{O}$, pressão expiratória máxima de $78,0(64,0-99,0) \mathrm{cmH}_{2} \mathrm{O}$ e percorreram $452,5(416,1-503,2)$ metros no teste de caminhada em seis minutos. CONCLUSÃO: Os pacientes cadastrados em lista de espera para o transplante renal do nosso estudo apresentaram uma boa condição físico funcional e cardiorrespiratória. $O$ tempo de diagnóstico da doença renal se correlacionou moderada e negativamente com a pressão expiratória máxima. $O$ tempo de permanência em lista de espera não se correlacionou com a resistência de membros inferiores, força muscular respiratória e capacidade funcional submáxima.

PALAVRAS-CHAVE: Transplante de rim. Insuficiência renal crônica. Avaliação em saúde. Fisioterapia. Teste de caminhada.
ABSTRACT | INTRODUCTION: For the treatment of chronic kidney disease, patients require hemodialysis or peritoneal dialysis while being on the kidney transplant waiting list. Despite advances in dialysis procedures, patients undergoing this treatment have low physical and functional capacity and comorbidities that influence the waiting time. OBJECTIVE: To describe the physical, anthropometric, and cardiorespiratory characteristics of patients on the kidney transplant waiting list, as well as to analyze the variables according to the time on the waiting list and time since disease diagnosis. MATERIALS AND METHODS: Cross-sectional, descriptive, and analytical study, submitted and approved by the Research Ethics Committee of UNIJUI under the document number 1.992.013. Eleven patients registered at the liuí Charity Hospital were evaluated for kidney disease and its treatment, time on waiting list, cardiovascular risk factors, lower limb muscle resistance, respiratory muscle strength, and submaximal functional capacity. RESULTS: From the eleven patients studied: the mean age was $51.9 \pm 11.4$ years; $54.5 \%$ were female, $45.5 \%$ had hypertensive renal disease, the time since disease diagnosis was 84.0 (48.0-180.0) months, $90.9 \%$ underwent hemodialysis with a median of $15.0(8.0-41.0)$ months, and were on the waiting list for 12.0 (6.0-32.0) months. The most prevalent cardiovascular risk factors were hypertension and sedentary lifestyle. Patients performed $17.0(16.0-22.0)$ repetitions in the 1 -minute sit-tostand test and walked 452.5 (416.1-503.2) meters in the sixminute walk test. The maximal inspiratory pressure was 62.0 $(50.0-80.0) \mathrm{cmH}_{2} \mathrm{O}$ and the maximal expiratory pressure was $78.0(64.0-99.0) \mathrm{cmH}_{2} \mathrm{O}$. CONCLUSION: In this study, patients enrolled in the kidney transplant waiting list presented good physical, functional, and cardiorespiratory conditions. The time since diagnosis of kidney disease was moderately and negatively correlated with maximal expiratory pressure. The time on the waiting list did not correlate with lower limb strength, respiratory muscle strength, and submaximal functional capacity.

KEYWORDS: Kidney transplantation. Chronic kidney failure. Health assessment. Physical therapy. Walking test. 


\section{Introdução}

A doença renal crônica é definida pela Sociedade Brasileira de Nefrologia como uma anormalidade estrutural ou funcional do rim, com ou sem diminuição da filtração glomerular' ${ }^{1}$ É um importante problema de saúde pública, com alto índice de mortalidade e presença de comorbidades ${ }^{2}$. Para o tratamento da doença renal crônica em fase avançada os pacientes necessitam de terapia de substituição renal dialítica, como hemodiálise e diálise peritoneal ou transplante renal. Atualmente há um aumento global do número de pacientes em diálise e também uma redução da taxa de mortalidade destes indivíduos ${ }^{3}$.

No Brasil existem 715 unidades ativas de diálise, onde 112.004 pacientes realizam tratamento dialítico, $20 \%$ das unidades de diálise estão localizadas na região sul do $\mathrm{Brasil}^{3}$. Segundo o Registro Brasileiro de Transplantes, atualmente 20.595 pacientes encontram-se em lista de espera para o transplante renal no Brasil. Destes, 839 pacientes estão inscritos no Rio Grande do Sul, sendo que no primeiro semestre de 2017 foram realizados no estado 423 transplantes, destes 403 foram realizados com órgão de doador cadáver e 20 com órgão de doador vivo ${ }^{4}$.

O fator idade e comorbidades são utilizados para cadastrar na lista de espera pacientes com menor risco de mortalidade 5 . Além disso, vários fatores estão relacionados ao tempo de permanência em lista de espera pelo transplante renal. Estudo realizado por Machado et al. ${ }^{6}$ em 2012 evidenciou que o tempo de espera pelo transplante foi menor em pacientes que não apresentaram nenhum tipo de comorbidade como causa da doença renal e naqueles pacientes que exerciam uma atividade profissional.

Apesar dos avanços nos procedimentos de diálise, os pacientes que fazem esse tratamento apresentam baixa tolerância ao exercício, o que leva ao descondicionamento físico, fator esse relacionado a comorbidades e que também pode estar associado ao tempo de permanência dos pacientes em lista de espera pelo transplante renal ${ }^{7-9}$. Vários estudos avaliaram a capacidade funcional de pacientes em tratamento dialítico ${ }^{7-9}$, porém nenhum deles estratificou esta análise de acordo com o tempo dos pacientes em lista de espera para $\circ$ transplante renal. Portanto, o objetivo deste estudo foi descrever as características físicas, antropométricas e cardiorrespiratórias de pacientes em lista de espera, que aguardavam para realizar o transplante renal, assim como, analisar as variáveis de acordo com o tempo de espera pelo transplante renal e tempo de doença renal crônica.

A partir dos dados obtidos neste estudo buscamos ressaltar a importância da avaliação físico funcional e cardiorrespiratória e da presença do fisioterapeuta na equipe de assistência à saúde do paciente com doença renal crônica a fim de nortear melhorias na assistência e reforçar a necessidade de intervenção física durante o período de realização de hemodiálise ou diálise peritoneal, e também no período pré e pós transplante renal.

\section{Materiais e métodos}

Estudo transversal, descritivo e analítico, recorte do Projeto institucional intitulado: "Acompanhamento de pacientes desde a lista de espera até pós transplante renal", que foi projetado de acordo com as Diretrizes e Normas Regulamentadoras de Pesquisas Envolvendo Seres Humanos segundo a Resolução do Conselho Nacional de Saúde $n^{\circ}$ 466/12, submetido e aprovado pelo Comitê de Ética em Pesquisa da UNIJUÍ parecer $\mathrm{n}^{\circ} 1.992 .013$; 63138116.9 .0000 .5350 .

A população em estudo foi constituída de pacientes que estavam em lista de espera para a realização do transplante renal no Hospital de Caridade de liuí - $\mathrm{HCl}$, os quais foram encaminhados pelos médicos nefrologistas do município de liuí/RS para a realização das avaliações. Foram incluídos na pesquisa participantes adultos de ambos os sexos e excluídos os indivíduos incapazes de entender e realizar os procedimentos dos testes. Durante - período do estudo, 24 pacientes estavam cadastrados em lista de espera para o transplante renal na região noroeste do estado do Rio Grande do Sul, destes, 12 pacientes (50\%) participaram do presente estudo, visto que o restante dos pacientes realizavam tratamento dialítico em outros hospitais da região. 
As avaliações foram realizadas no setor de Nefrologia do $\mathrm{HCl}$, em dia agendado previamente, no mesmo dia em que os pacientes tinham consulta médica com $\circ$ nefrologista. Os participantes da pesquisa foram submetidos ao protocolo de avaliação, conforme descrito a seguir.

A partir da entrevista direta com o paciente foram coletados dados demográficos e clínicos como: a causa da doença renal, tempo de diagnóstico da doença, presença de comorbidades, fatores de risco para doenças cardiovasculares, tipo de tratamento dialítico, o tempo de realização e de inserção na lista de espera.

Foram avaliadas as seguintes medidas antropométricas: peso corporal em quilogramas $(\mathrm{kg})$; estatura medida em metros $(\mathrm{m})$; circunferência abdominal medida em centímetros $(\mathrm{cm})$ aferida na distância média entre a última costela flutuante e a crista ilíaca. Para isso foi utilizada fita métrica com padrão flexível e inextensível, com definição de medida de 0,1 cm. $O$ índice de massa corporal (IMC) foi calculado pela razão entre o peso e o quadrado da altura $\left(\mathrm{kg} / \mathrm{m}^{2}\right)$.

Para determinar o índice de conicidade foram utilizadas as medidas de peso corporal, estatura e circunferência abdominal utilizando-se a equação matemática de Valdez ${ }^{10}$, que foi calculada utilizando - programa da Microsoft Excel. Para determinar risco coronariano elevado foi utilizado os pontos de corte de $\geq 1,25$ para homens e $\geq 1,18$ para mulheres com base no estudo de Pitanga e Lessa (2004):

$$
\text { Índice de Conicidade }=\frac{\text { Circunferência abdominal }(\mathrm{cm})}{0,109 \times \frac{\sqrt{\text { Peso corporal }(\mathrm{Kg})}}{\text { Estatura }(\mathrm{m})}}
$$

Para avaliar a resistência muscular de membros inferiores foi utilizado o teste de sentar e levantar em um minuto (TSL1'), para o qual o indivíduo foi orientado a sentar-se em uma cadeira com as costas repousando sobre o encosto da mesma, e logo após levantar-se, sem apoiar os braços, estendendo os joelhos e na sequência sentar-se novamente tocando as costas no encosto da cadeira. $O$ indivíduo deveria realizar o máximo de elevações possíveis em um minuto ${ }^{11}$.
A força muscular respiratória foi determinada através da aferição da pressão inspiratória máxima (PImáx) e pressão expiratória máxima (PEmáx), sendo utilizado o manovacuômetro digital modelo MVD-300 (Microhard System, Globalmed, Porto Alegre, Brasil). Durante o procedimento os indivíduos permaneceram sentados em repouso, com o tronco ereto e as narinas ocluídas com clipe nasal para evitar o escape de ar. A PEmáx foi mensurada após - paciente expirar da capacidade pulmonar total até o volume residual, com consequente esforço expiratório máximo, neste momento foi ocluído o orifício existente no bocal. Enquanto, que a Plmáx foi obtida após o paciente inspirar do volume residual até a capacidade pulmonar total, gerando um esforço inspiratório máximo, sendo o orifício do bocal ocluído da mesma maneira da aferição da PEmáx. Os maiores valores de PImáx e de PEmáx, expressos em $\mathrm{cmH}_{2} \mathrm{O}$, foram registrados, não sendo este maior que $10 \%$ do segundo maior valor ${ }^{12}$. Foram realizados cálculos do previsto para tais variáveis $^{12}$. Quando o indivíduo atingir um valor menor ou igual a $70 \%$ do previsto é considerado com força muscular diminuída.

A capacidade funcional submáxima foi analisada através do teste de caminhada em seis minutos (TC6'), realizado segundo as recomendações da American Thoracic Society ${ }^{13}$, no qual foi mensurada a maior distância que 0 indivíduo foi capaz de percorrer num intervalo de tempo fixo de seis minutos. Os indivíduos foram orientados quanto a realização do teste, conforme protocolo. O cálculo da distância percorrida prevista (em metros) para o indivíduo foi realizado por meio das fórmulas de Enright e Sherrill ${ }^{14}$.

Para o processamento dos dados foi utilizado o programa estatístico Statistical Package for Social Science - SPSS (versão 18.0, Chigago, IL, EUA). As variáveis qualitativas foram apresentadas por meio de frequências e porcentagens e as quantitativas por média e desvio padrão (média \pm DP) ou mediana e intervalo interquartil, conforme apropriado. O coeficiente de correlação de Spearman foi empregado para avaliar a correlação entre TSL1', PImax, PEmax e TC6' com o tempo de espera pelo transplante e tempo de DRC, conforme a distribuição das variáveis. Considerou-se estatisticamente significativo $p<0,05$. Todos os testes foram aplicados com intervalo de confiança de $95 \%$. 


\section{Resultados}

Do total de 12 pacientes inscritos na lista de espera para o transplante renal e em tratamento dialítico no $\mathrm{HCl}, 11$ pacientes foram incluídos no estudo, sendo um excluído devido estar em isolamento de contato durante o período de coleta de dados. A média de idade foi de $51,9 \pm 11,4$ anos, sendo $54,5 \%$ do sexo feminino, Tabela 1 .

Tabela 1. Caracterização clínica da amostra de pacientes em lista de espera para o transplante renal, 2017

\begin{tabular}{|c|c|}
\hline Variáveis & $n=11$ \\
\hline $\begin{array}{l}\text { Idade (anos) } \\
\text { Etiologia da Doença Renal n (\%) }\end{array}$ & $51,9 \pm 11,4$ \\
\hline Hipertensiva & $5(45,5)$ \\
\hline Doença Policística & $3(27,3)$ \\
\hline Glomerulonefrite & $2(18,2)$ \\
\hline Agenesia Renal Unilateral & $1(9,1)$ \\
\hline Tempo de doença renal (meses) & $84,0(48,0-180,0)$ \\
\hline \multicolumn{2}{|l|}{ Tratamento Dialítico n (\%) } \\
\hline Hemodiálise & $10(90,9)$ \\
\hline Diálise Peritoneal & $1(9,1)$ \\
\hline Tempo de tratamento dialítico (meses) & $15,0(8,0-41,0)$ \\
\hline Tempo de permanência em lista de espera (meses) & $12,0(6,0-32,0)$ \\
\hline \multicolumn{2}{|l|}{ Fatores de risco cardiovasculares $\mathbf{n}(\%)$} \\
\hline Hipertensão Arterial Sistêmica & $10(90,9)$ \\
\hline Dislipidemia & $1(9,1)$ \\
\hline Sedentarismo & $8(72,7)$ \\
\hline Estresse & $4(36,4)$ \\
\hline Idade $>60$ anos & $3(27,3)$ \\
\hline Sexo masculino & $5(45,5)$ \\
\hline Obesidade & $2(18,2)$ \\
\hline História familiar & $4(36,4)$ \\
\hline \multicolumn{2}{|l|}{ Medidas Antropométricas } \\
\hline$I M C\left(K g / m^{2}\right) F / M$ & $27,6 \pm 5,8 / 25,5 \pm 5,0$ \\
\hline Circunferência abdominal $(\mathrm{cm}) \mathrm{F} / \mathrm{M}$ & $97,6 \pm 15,9 / 95,4 \pm 16,7$ \\
\hline Índice de Conicidade F / M & $1,32 \pm 0,14 / 1,31 \pm 0,12$ \\
\hline
\end{tabular}

Na Tabela 2 estão apresentados os dados da avaliação físico funcional e cardiorrespiratória dos pacientes em lista de espera para o transplante renal.

Tabela 2. Avaliação físico funcional e cardiorrespiratória de pacientes em lista de espera para o transplante renal, 2017

\begin{tabular}{|c|c|}
\hline Variáveis & $n=11$ \\
\hline $\mathrm{N}^{\circ}$ Repetições no TSL1' & $17,0(16,0-22,0)$ \\
\hline PImax $\left(\mathrm{cmH}_{2} \mathrm{O}\right)$ & $62,0(50,0-80,0)$ \\
\hline PImax prevista $\left(\mathrm{cmH}_{2} \mathrm{O}\right)$ & $87,9(86,0-117,0)$ \\
\hline$\%$ do previsto na PImax & $73,0(44,0-86,4)$ \\
\hline PEmax $\left(\mathrm{cmH}_{2} \mathrm{O}\right)$ & $78,0(64,0-99,0)$ \\
\hline PEmax prevista $\left(\mathrm{cmH}_{2} \mathrm{O}\right)$ & $87,1(84,7-116,7)$ \\
\hline$\%$ do previsto na PEmax & $93,0(56,0-109,0)$ \\
\hline 'Distância percorrida no TC6' (metros) & $452,5(416,1-503,2)$ \\
\hline Distância prevista percorrida no TC6' (metros) & $573,6(532,3-593,0)$ \\
\hline \% alcançado do previsto TC6' (metros) & $81,6(71,7-87,5)$ \\
\hline
\end{tabular}


Foi verificada a correlação do tempo de permanência em lista de espera para o transplante renal e do tempo de diagnóstico da doença renal crônica com as variáveis físico funcionais e cardiorrespiratórias. Observouse correlação moderada negativa entre o tempo de diagnóstico da doença renal e a pressão expiratória máxima $(r=-0,624, p=0,040)$, como apresentado na Tabela 3.

Tabela 3. Correlação do tempo de doença renal e tempo em lista de espera para o transplante renal com o TC6', Plmáx, PEmáx e TSL1', 2017

\begin{tabular}{|c|c|c|c|c|c|}
\hline & & DTC6' & PImáx & PEmáx & TSLI' \\
\hline \multicolumn{6}{|l|}{ Variáveis } \\
\hline \multirow{2}{*}{$\begin{array}{l}\text { Tempo de diagnóstico da doença } \\
\text { renal }\end{array}$} & $\mathbf{r}$ & 0,091 & $-0,475$ & $-0,624^{*}$ & $-0,119$ \\
\hline & $\mathbf{p}$ & 0,802 & 0,140 & 0,040 & 0,728 \\
\hline \multirow{2}{*}{$\begin{array}{l}\text { Tempo em lista de espera para o } \\
\text { transplante renal }\end{array}$} & $r$ & $-0,018$ & 0,237 & 0,100 & $-0,586$ \\
\hline & $\mathbf{p}$ & 0,960 & 0,483 & 0,770 & 0,058 \\
\hline
\end{tabular}

\section{Discussão}

A principal causa de doença renal dos pacientes cadastrados em lista de espera para o transplante renal participantes do presente estudo foi de origem hipertensiva, a mediana do tempo de diagnóstico de doença renal foi de 84,0 meses e do tempo de permanência em lista de espera para o transplante foi de 12 meses. Na avaliação físico funcional e cardiorrespiratória, os pacientes apresentaram um bom desempenho nos testes atingindo acima de $70 \%$ do seu previsto, sendo observado correlação moderada negativa entre o tempo de diagnóstico da doença renal e a pressão expiratória máxima.

A maioria dos pacientes em lista de espera avaliados em nosso estudo realizavam a hemodiálise como terapia de substituição renal, sendo que houve um transplante com doador vivo durante o período de coleta de dados. Nossos dados estão de acordo com os dados de estudos prévios, onde dentre as terapias de substituição renal a mais utilizada é a hemodiálise ${ }^{3}$, porém o transplante renal é a que oferece melhor sobrevida e qualidade de vida ${ }^{19}$.

Emnosso estudo o tempomédio de início do tratamento dialítico foi de 27 meses. Estudos que avaliaram pacientes em hemodiálise não cadastrados em lista de espera encontraram tempo de realização de terapia de substituição renal superior $7,9,20$. Estudo realizado em São Paulo encontrou um tempo de permanência em lista de espera de 63 meses, tempo superior à mediana encontrada em nosso estudo ${ }^{15}$. Portanto, Batista et al $^{15}$ reforçam a importância de realizar a inscrição dos pacientes em lista de espera para o transplante renal concomitantemente ao início da terapia de substituição renal, pois o tempo em diálise pode influenciar negativamente na condição clínica dos pacientes. Segundo Rigoni et al ${ }^{21}$ o tempo de permanência em lista de espera é menor para pacientes que realizam dialise peritoneal.

A média de idade dos pacientes cadastrados em lista de espera do nosso estudo foi semelhante à encontrada em um estudo recente realizado em São Paulo ${ }^{15}$. Na Noruega, a média de idade dos pacientes em lista de espera foi de 71,1 anos, ${ }^{16} \mathrm{e}$ nos Estados Unidos está sendo realizado com maior frequência transplantes com pacientes idosos acima de 65 anos $^{17}$. Tais dados sugerem que a população brasileira está desenvolvendo mais precocemente a doença renal ou que a progressão da doença está ocorrendo mais rapidamente na nossa população.

Aproximadamente metade dos pacientes avaliados em nosso estudo são do sexo feminino. Machado et $a l^{6}$ encontrou uma frequência de $37,7 \%$ e Batista et $\mathrm{al}^{15}$ encontrou uma frequência de $40,4 \%$. A doença 
renal de origem hipertensiva foi a mais frequente, corroborando com os dados de alguns estudos $3,7,15$. A obesidade também tem sido descrita como causa de doença renal devido à hiperlipidemia, aumento do estresse oxidativo, aumento da ingestão de sal, e ativação do sistema nervoso simpático ${ }^{18}$. Em nosso estudo $18,2 \%$ dos pacientes foram classificados como obesos e 36,4\% como sobrepeso. Portanto, a prevalência de obesidade encontrada em nosso estudo foi maior do que a reportada na literatura, onde $37 \%$ dos pacientes são classificados acima do peso ${ }^{3}$.

Quanto aos fatores de risco cardiovasculares, os mais prevalentes foram hipertensão arterial sistêmica e sedentarismo, confirmando dados encontrados na literatura ${ }^{7,9}$. Além dos valores antropométricos elevados de índice de massa corporal, circunferência abdominal e índice de conicidade ${ }^{22}$. Na avaliação deste índice foram adotados os pontos de corte de $\geq 1,25$ e $\geq 1,18$ para homens e mulheres, respectivamente, para determinar risco coronariano elevado, com base no estudo de Pitanga e Lessa ${ }^{23}$, portanto, em nosso estudo, $81,8 \%$ dos pacientes apresentaram risco coronariano elevado. Szabo et $\mathrm{al}^{24}$ descreveu que a maioria dos pacientes em lista de espera são identificados como baixo risco. Segundo Leford et $a^{25}$ a presença de mais de três fatores de riscos cardiovasculares diminuiu em $49 \%$ a chance de inscrição em lista de espera.

Apesar dos fatores de risco cardiovascular, os pacientes do presente estudo apresentaram um bom desempenho na avaliação físico funcional e cardiorrespiratória. Pacientes que realizam hemodiálise e não estão cadastrados em lista de espera apresentaram menor desempenho na avaliação da força muscular respiratória ${ }^{7,8}$. Pacientes com doença renal crônica em hemodiálise ${ }^{7}$ e que ainda não iniciaram o tratamento dialítico ${ }^{9}$ também apresentaram desempenho funcional inferior no TC6'. Portanto, sugere-se que estas variáveis são fatores associados tão importantes quando idade e comorbidades que podem ser utilizados como critérios para cadastrar pacientes em lista de espera para o transplante.

Nosso estudo apresenta limitações potenciais. Primeiro, o pequeno tamanho da amostra e o fato dos participantes do estudo serem de um único centro, limita a generalização dos dados. Segundo, a inexistência de um grupo controle limitou a comparabilidade dos resultados. Por último, os dados da avaliação físico funcional e cardiorrespiratória foram analisados a partir dos valores preditos por meio de fórmulas que podem não refletir a realidade da população de pacientes com insuficiência renal crônica que realizam tratamento dialítico no Brasil.

\section{Conclusão}

Apesar da presença elevada de fatores de risco cardiovascular e risco coronariano elevado os pacientes cadastrados em lista de espera para o transplante renal do nosso estudo apresentaram uma boa condição físico funcional e cardiorrespiratória. $O$ tempo de diagnóstico da doença renal se correlacionou moderada e negativamente com a pressão expiratória máxima. O tempo de permanência em lista de espera não se correlacionou com a resistência de membros inferiores, força muscular respiratória e capacidade funcional submáxima. Dessa forma, sugere-se que as variáveis físico funcionais e cardiorrespiratórias são fatores associados tão importantes quando idade e comorbidades que podem ser utilizados como critério para cadastrar pacientes em lista de espera para o transplante. Sendo assim, ressalta-se a importância da inserção da assistência fisioterapêutica para a avaliação, prevenção e reabilitação física destes pacientes. Há necessidade de mais estudos nesta área com maior número amostral.

\section{Contribuições das autoras}

Daniela da Silva Martins participou da concepção, delineamento, busca e análise estatística dos dados da pesquisa, interpretação dos resultados, redação do artigo científico. Paula Caitano Fontela participou da análise estatística dos dados da pesquisa e da redação do artigo científico. Maria Leocadia Bernardes do Amaral Padilha participou da concepção e redação do artigo científico. Eliane Roseli Winkelmann participou da concepção, delineamento, análise estatística dos dados da pesquisa, interpretação dos resultados, redação e encaminhamento do artigo científico.

\section{Conflitos de interesses}

Nenhum conflito financeiro, legal ou político envolvendo terceiros (governo, empresas e fundações privadas, etc.) foi declarado para nenhum aspecto do trabalho submetido (incluindo mas não limitandose a subvenções e financiamentos, conselho consultivo, desenho de estudo, preparação de manuscrito, análise estatística, etc). 


\section{Referências}

1. Sociedade Brasileira de Nefrologia. Censo de diálise, 2013 [Internet]. 2013. Disponível em: https://sbn.org.br/ resultados-do-censo-2013/

2. de Moura L, Andrade SSCA, Malta DC, Pereira CA, Passos JEF. Prevalence of self-reported chronic kidney disease in Brazil: National Health Survey of 2013. Rev Bras Epidemiol. 2015;18(supl 2):181-91. doi: 10.1590/1980$\underline{5497201500060016}$

3. Sesso RC, Lopes AA, Thomé FS, Lugon JR, Martins CT. Inquérito Brasileiro de Diálise Crônica 2014. J Bras Nefrol. 2016;38(1):54-61. doi: 10.5935/0101-2800.20160009

4. Associação Brasileira de Transplante de Órgãos. Registro Brasileiro de Transplante. Dados numéricos da doação de órgãos e transplantes realizados por estado e instituição no período: Janeiro / Setembro - 2017 [Internet]. 2017. Disponível em: http://www.abto.org.br/abtov03/Upload/ file/RBT/2017/rbttrim3-leitura.pdf

5. Ponticelli C, Podesta MA, Graziani G. Renal transplantation in elderly patients. How to select the candidates to the waiting list? Transplant Rev (Orlando). 2014;28(4):188-92. doi: $10.1016 /$ i.trre.2014.07.001

6. Machado EL, Gomes IC, de Assis Acurcio F, César CC, de Mattos Almeida MC, Cherchiglia ML. Fatores associados ao tempo de espera e ao acesso ao transplante renal em Belo Horizonte, Minas Gerais. Cad Saúde Pública. 2012 ;28(1 2):2315-26. doi: 10.1590/S0102$\underline{311 \times 2012001400010}$

7. Posser SR, Cecagno-Zanini SC, Piovesan F, Leguisamo $\mathrm{CP}$. Functional capacity, pulmonary and respiratory muscle strength in individuals undergoing hemodialysis. Fisioter Mov. 2016;29(2):343-50. doi: 10.1590/0103-5150.029.002. $\underline{\mathrm{A} 013}$

8. Roxo RS, Xavier VB, Miorin LA, Magalhães AO, Sens YA, Alves VL. Impact of neuromuscular electrical stimulation on functional capacity of patients with chronic kidney disease on hemodialysis. J Braz Nephrol. 2016;38(3):344-350. doi: 10.5935/0101-2800.20160052

9. Fassbinder TRC, Winkelmann ER, Schneider J, Wendland J, de Oliveira OB. Capacidade funcional e qualidade de vida de pacientes com doença renal crônica pré-dialítica e em hemodiálise - Um estudo transversal. J bras nefrol. 2015;37(1):47-54. doi: 10.5935/0101-2800.20150008

10. Valdez R. A simple model-based index of abdominal adiposity. J Clin Epidemiol. 1991;44(9):955-6.

11. de Araújo CGS. Teste de sentar-levantar: apresentação de um procedimento para avaliação em Medicina do Exercício e do Esporte. Rev Bras Med Esporte. 1999;5(5):179-82. doi: 10.1590/S1517. 86921999000500004
12. Neder JA, Nery LE. Fisiologia clínica do exercício-teoria e prática: variáveis e parâmetros obtidos no teste de exercício cardiorespiratório. São Paulo: Artes Médicas;2003.

13. Holland AE, Spruit MA, Troosters T, Puhan MA, Pepin $V$, Saey $D$ et al. An official European Respiratory Society/ American Thoracic Society technical standard: field walking tests in chronic respiratory disease. Eur Respir J. 2014;44(6):1428-46. doi: $10.1183 / 09031936.00150314$

14. Enright PL, Sherrill DL. Reference equations for the sixminute walk in healthy adults. Am J Respir Crit Care Med. 1998;158:1384-7. doi: 10.1164/ajrccm.158.5.9710086

15. Batista CMM, Moreira RSL, Pessoa JLE, Ferraz AS, Roza BdA. Perfil epidemiológico dos pacientes em lista de espera para o transplante renal. Acta Paul Enferm. 2017;30(3):2806. doi: $10.1590 / 1982-0194201700042$

16. Lonning K, Midtvedt K, Bernklev T, Brunborg C, Andersen $\mathrm{MH}$, von der Lippe $\mathrm{N}$ et al. Changes in health related quality of life in older candidates waiting for kidney transplantation. Nephrology (Carlton). 2017. doi: 10.1111/nep.13117

17. Concepcion BP, Forbes RC, Schaefer HM. Older candidates for kidney transplantation: Who to refer and what to expect? World J Transplant. 2016;6(4):650-7. doi: 10.5500/wit.v6.i4.650

18. Chandra A, Biersmith $M$, Tolouian R. Obesity and kidney protection. Journal of nephropathology. 2014;3(3):91-7. doi: 10.12860/inp.2014.18

19. Czyzewski L, Sanko-Resmer J, Wyzgal J, Kurowski A. Assessment of health-related quality of life of patients after kidney transplantation in comparison with hemodialysis and peritoneal dialysis. Ann Transplant. 2014;19:576-85. doi: $\underline{10.12659 / \text { AOT.891265 }}$

20. Fructuoso M, Castro R, Oliveira L, Prata C, Morgado T. Quality of life in chronic kidney disease. Nefrologia. $2011 ; 31(1): 91-6$. doi:10.3265/Nefrologia.pre2010. $\underline{\text { Jul. } 10483}$

21. Rigoni $M$, Torri E, Nollo G, Zarantonello D, Laudon $A$, Sottini $L$ et al. Survival and time-to-transplantation of peritoneal dialysis versus hemodialysis for end-stage renal disease patients: competing-risks regression model in a single Italian center experience. J Nephrol. 2017;30(3):441-7. doi: 10.1007/s40620-016-0366-6

22. Alves LF, Abreu TTd, Neves NCS, Morais FAd, Rosiany IL, Júnior WVO et al. Prevalence of chronic kidney disease in a city of southeast Brazil. J Bras Nefrol. 2017;39(2):126-34. doi: $10.5935 / 0101-2800.20170030$

23. Pitanga FJG, Lessa I. Sensibilidade e especificidade do índice de conicidade como discriminador do risco coronariano de adultos em Salvador, Brasil. Rev Bras Epidemiol. 2004. 7(3). doi: 10.1590/S1415-790X2004000300004 
24. Szabo RP, Varga I, Balla J, Zsom L, Nemes

B. Cardiovascular Screening and Management

Among Kidney Transplant Candidates in Hungary.

Transplant Proc. 2015;47(7):2192-5. doi: 10.1016/i.

transproceed.2015.07.018

25. Lefort $M$, Vigneau $C$, Laurent $A$, Lebbah $S$, Le Meur $N$, Jais JP et al. Facilitating access to the renal transplant waiting list does not increase the number of transplantations: comparative study of two French regions. Clin Kidney J. 2016;9(6):849-57. doi: $10.1093 / \mathrm{cki} / \mathrm{sfw} 078$ 